\title{
Death by Delirium: A 71-Year-Old Male with Poor Post-operative Recovery
}

William Bradford, MD

\section{INTRODUCTION}

Delirium is an acute decline in attention and cognition not better explained by another medical condition. ${ }^{1}$ It is multifactorial in origin, with risk factors including advanced age, male sex, baseline decreased cognitive status, sensory impairment, poor baseline functional status, polypharmacy, and multiple comorbid conditions. Acute precipitants for delirium include administration of psychoactive medications (particularly benzodiazepines, narcotics, anticholinergic medications, and general anesthesia), sleep deprivation, major surgery, and new illness or worsening of current illness. ${ }^{1}$ Delirium accounts for some $49 \%$ of all hospital days in older patients, increases rates of mortality and morbidity, and is associated with lasting cognitive impairment after discharge, even in younger patients. ${ }^{2}$

\section{CASE PRESENTATION}

Mr. M was a 71-year-old male Vietnam-War veteran living independently and working as a standardized patient at a local medical college. The patient had a past medical history of cirrhosis due to nonalcoholic steatohepatitis (NASH), multiple sclerosis (MS), atrial fibrillation (AF), and diverticulosis. He presented as a transfer from an outside hospital for management of decompensated NASH cirrhosis precipitated by a mechanical fall, resulting in a right hip replacement eleven days prior. He was admitted to an inpatient hepatology service and shortly after admission, he was noted to have altered mental status. Admission blood cultures were positive for Klebsiella pneumoniae and purulent drainage was noted from the hip incision. The patient was taken to the operating room by orthopedic surgery for hip replacement washout and revision and transferred to the surgical intensive care unit (SICU) postoperatively for hypoxic respiratory failure requiring intubation. After an 8 day course in the SICU marked by persistent delirium, the patient was transferred back to the hepatology service for further management.

On arrival to the hepatology service, Mr. M had moderate global weakness, severe dysphagia requiring tube feeds via a dobhoff tube (DHT), and recurrent bouts of symptomatic AF with rapid ventricular response. The patient was maintained on ciprofloxacin for suppression of his prosthetic joint infection. His course over the ensuing days was marked by episodes of disorientation, vivid visual and auditory hallucinations, and alternating somnolence and agitation. His hospital course was further complicated by bilateral lower extremity deep vein thromboses, persistent severe dysphagia, post-traumatic stress disorder with flashbacks, periodic mild hypernatremia, and poor tolerance of physical therapy. He had worsening of his hallucinations, inability to follow commands, and decreased orientation after an EGD for banding of esophageal varices. These symptoms improved slightly after his home baclofen (for multiple sclerosis) was tapered and ceftriaxone was substituted for ciprofloxacin. Head magnetic resonance (MR) imaging, $M R$ angiography and multiple computed tomography (CT) scans showed no evidence of an acute intracranial process or major worsening of his known MS lesions. His blood urea nitrogen (BUN) was within normal limits, and he was maintained on lactulose and rifaximin with stool output at or above goal 3-4/day throughout his stay. On hospital day 53, 39 days after his transfer from the SICU to the floor, he developed lactic acidosis, severe acute kidney injury, and became acutely hypoxic with increased work of breathing. He was offered intubation and further workup for his severe sepsis; however, his decision-maker opted for hospice.

\section{DIFFERENTIAL DIAGNOSIS}

Mr. M suffered discrete episodes of altered mental status that were precipitated by anesthesia, sleep deprivation due to late lab draws, nighttime administration of ciprofloxacin and baclofen, and known triggers of his post-traumatic stress disorder (including hospital helicopter landings and thirst). These episodes decreased in frequency with adequate sleep, reorientation measures, control of his thirst, use of atypical antipsychotics, and removal of a fecal management system. His ammonia was elevated initially at 49, at which point he was empirically started on lactulose and rifaximin with stool output at or above goal. He was englycemic throughout his stay. The patient had no kidney disease or elevated BUN to suggest uremia. Exhaustive brain imaging did not reveal any significant intracranial pathology. Comprehensive infectious workup found no new infection or recurrence of his previous joint infection. Given his critical illness, waxing and waning mental status, and lack of improvement despite correction of other identifiable causes, the diagnosis of delirium was made. 


\section{OUTCOME AND FOLLOW-UP}

The patient expired two days after transfer to inpatient hospice.

\section{DISCUSSION}

Mr. M's unfortunate case illustrates prolonged delirium leading to a cascade of complications that ultimately led to what was likely a hospital-acquired infection resulting in his death. This case highlights the importance of early recognition and aggressive treatment of delirium in hospitalized elderly patients. Although Mr. M predominantly displayed hyperactive delirium, it is estimated that some $75 \%$ of delirium cases are the more insidious hypoactive form, which is associated with worse outcomes, possibly because it more often goes undiagnosed and untreated. ${ }^{3}$ As the front-line provider for inpatients, the resident physician is able to have a major impact on the recognition and treatment of delirium.

Recognition of delirium begins with clinical suspicion based on risk factors. A bedside diagnostic test, such as the Confusion Assessment Method (Figure 1A), should follow. ${ }^{1}$ If the diagnosis of delirium is confirmed, the clinician should perform a thorough evaluation for the underlying cause. The DELIRIUM mnemonic provides a useful approach for this evaluation (Figure 1B). ${ }^{1}$ The cornerstone of delirium management is well-integrated care by all members of the healthcare team as well as the patient's family to identify and address all modifiable contributors. Physical restraints, although frequently utilized, are actually associated with increased risk of

\section{Figure 1A: The Confusion Assessment Method} for diagnosing delirium

To diagnose delirium, features 1 and 2 and either 3 or 4 are required

1. Acute change in mental status with a fluctuating course

2. Inattention

3. Disorganized thinking

4. Altered level of consciousness

\section{Figure 1B: The DELIRIUM mnemonic for} underlying causes of delirium

\section{Drugs}

Electrolyte disturbances

Lack of drugs

Infection

Reduced sensory input

Intracranial etiologies

Urinary and fecal disorders

Myocardial and pulmonary disorderss injury and persistence of delirium after discharge. ${ }^{4}$ Their use should be reduced or eliminated on the general wards but may be necessary in the ICU to prevent dislodgement of lines and tubes. Use of a sitter should be considered in lieu of restraints. Pharmacologic treatment with antipsychotics may be required for patients with distressing perceptual disturbances or delusional thoughts but should not be employed without attention to potential underlying reversible causes.

Prevention is the best approach to delirium, with the key components of the prevention strategy established in a 1999 study which showed that reorientation, non-pharmacologic sleep protocol, getting the patient out of bed, encouraging the use of glasses/hearing aids, and encouraging fluid intake result in lower rates of delirium in hospitalized elderly. ${ }^{5}$ Some studies also show benefits for elderly patients who receive geriatric consultation early in their hospital stay. More aggressive prevention, especially closer attention to hydration status and more attention to preventing nocturnal awakenings early in his stay, might have prevented such a tragic outcome for Mr. $M$ and other patients like him.

\section{KEY POINTS}

- Delirium, especially the hypoactive form, is an underrecognized and preventable complication of hospitalization in elderly patients.

- Advanced age, male sex, baseline cognitive impairment, polypharmacy, and multiple comorbidities are risk factors for development of delirium.

- Reorientation, institution of a non-pharmacologic sleep protocol, getting the patient out of bed, encouraging the use of glasses/hearing aids, and encouraging fluid intake result in lower rates of delirium in hospitalized elderly.

- Antipsychotic medications should only be used when necessary to prevent harm to the patient and should be initiated at a very low dose $10.25 \mathrm{mg} \mathrm{IV}$ is the recommended starting dose of haloperidol).

\section{REFERENCES}

1. Marantonio, E. Delirium in Hospitalized Older Adults. N Engl J Med. 2017:377:1456-66.

2. Pandharipande PP, Girard TD, Jackson JC, et al. Long-term cognitive impairment after critical illness. N Engl J Med. 2013;369:1306-16.

3. Kiely DK, Jones RN, Bergmann MA, Marcantonio ER. Association between psychomotor activity delirium subtypes and mortality among newly admitted post-acute facility patients. J Gerontol A Biol Sci Med Sci. 2007;62:174-179.

4. Inouye SK, Zhang Y, Jones RN, Kiely DK, Yang F, Marcantonio ER. Risk factors for delirium at discharge: development and validation of a predictive model. Arch Intern Med. 2007;167:1406-1413.

5. Inouye SK, Bogardus ST Jr, Charpentier PA, et al. A multicomponent intervention to prevent delirium in hospitalized older patients. N Engl J Med. 1999;340:669-76. 